\title{
Internal consistency for embedding complexity
}

\author{
Sy-David Friedman and Katherine Thompson
}

December 18, 2007

\begin{abstract}
In a previous paper with M. Džamonja, class forcings were given which fixed the complexity (a universality covering number) for certain types of structures of size $\lambda$ together with the value of $2^{\lambda}$ for every regular $\lambda$. As part of a programme for examining when such global results can be true in an inner model, we build generics for these class forcings. ${ }^{1}$
\end{abstract}

\section{Introduction}

The internal consistency programme was introduced in [6]. This programme aims to determine which consistent statements of set theory are in fact internally consistent, i.e., true in an inner model, assuming the existence of large cardinals. One of the main advantages of internal consistency is that this rules out consistent statements, such as the nonexistence of transitive set models of ZFC, which can only hold in universes which are incompatible with $V$.

There are many consistency results achieved using forcing which are not yet known to be obtainable via inner models, since internal consistency results are harder to achieve. To obtain any consistency result, one typically makes

\footnotetext{
${ }^{1}$ This research was supported by the Austrian Science Fund (FWF), Grants P 16334NO5 and P 16790-NO4.
} 
use of a generic filter. To obtain consistency results using set-forcing, one may assume that the starting model is countable and therefore the existence of a generic is trivial. To achieve internal consistency, one needs an inner model with all the ordinals of the ambient universe, therefore one cannot restrict to a countable submodel.

The question then is how to build a generic, if one even exists. The methods used to do this require new techniques which are mathematically of independent interest. Since there are strong connections between work done in large cardinal theory and in internal consistency (see e.g. [6], [2]), these techniques can be applied in the context of other questions involving large cardinals (see e.g. [8]).

For more about this programme, including a discussion of some of the results that have been obtained within it, we refer the reader to [6].

A number of the early internal consistency result below $0^{\#}$ are discussed in Chapter 3 of [5]. These result use either reverse Easton or forward Easton forcing methods. An example of the latter is due to Jensen [1], who showed that in $L\left[0^{\#}\right]$ there is a real which is class-generic but not set-generic over $L$. All of these early results however only produce models of GCH.

The first example of an internal consistency result where GCH fails appears in [7]. In that paper, Easton's theorem ([4]) is examined in the internal consistency context, and it is shown that any (parameter-free) $L$-definable Easton function can be realised as the generalised continuum function $\kappa \mapsto 2^{\kappa}$ in an inner model of $L\left[0^{\#}\right]$. Such a result cannot be obtained using the forcing that Easton introduced, the Easton product. Instead, [7] introduces a reverse Easton iteration of Easton products, and then shows how to obtain a generic over $L$ for this class-forcing using the Silver indiscernibles. A generic for the forcing up to and including $i$, denoted $G(\leq i)$, is constructed by induction on the Silver indiscernible $i$; the main task is to choose these to cohere in the sense that $G(\leq j)$ contains the image of $G(\leq i)$ under the natural embedding $\pi_{i j}: L \rightarrow L$ obtained by "shifting" the class $I$ of indiscernibles onto $I \backslash[i, j)$. The technique of "generic modification" is used in order to modify a given generic $G^{\prime}(\leq j)$ to a generic $G(\leq j)$ with the desired coherence property.

Our work in the present paper extends that of [7] in two new directions. First, we show how to construct generics for forcings for which the technique 
of generic modification cannot be used. Instead, we construct our generics directly so as to cohere. Second, we establish the first internal consistency result (section 5) that handles a reverse Easton iteration of forcings whose building blocks are not products, as in [7], but which are themselves iterations. This presents some significant new challenges that we meet using special dense sets of conditions and elementary submodels.

The context of the present paper is that of embedding complexity. However, the techniques we introduce here for the construction of generics have the potential of application to many other contexts. We discuss this point further in the final section.

In order to explain our results on embedding complexity, we begin with the basic universality definitions. An embedding (also called weak embedding) for ordered sets is an injective order-preserving map. A strong embedding is an embedding which also preserves incomparability. The range of a strong embedding is an isomorphic copy of the order in the domain. For graphs, an embedding is an injective function which preserves edges and a strong embedding is an injective function whose range is an induced subgraph.

Given a set $\mathcal{A}(\lambda)$ of structures each of size $\lambda$, a (strong) universal model for $\mathcal{A}(\lambda)$ is an element of $\mathcal{A}(\lambda)$ which (strongly) embeds all other structures in $\mathcal{A}(\lambda)$. If there does not exist a universal model for $\mathcal{A}(\lambda)$, then we consider its complexity, or the smallest size of a subfamily of $\mathcal{A}(\lambda)$ which embeds the rest. This subfamily of structures is called a universal family. All of these notions have weak and strong counterparts depending on the type of embedding used.

In this paper, $\lambda, \kappa$ will be regular cardinals such that $\lambda \geq \kappa \geq \aleph_{0}$. Let $\mathcal{C}(\lambda, \kappa)$ be the set (of representatives under isomorphism) of all posets of size $\lambda$ which omit $\kappa$-chains. Denote by $G(\lambda, \kappa)$ the set of all graphs of size $\lambda$ which omit $\kappa$-cliques.

In the paper [3], two class forcing results were given. One says that the weak complexity for $\mathcal{C}(\lambda, \kappa)$ (or $G(\lambda, \kappa)$ ) can be $2^{\lambda}$ for any "reasonable" value of $2^{\lambda}$ and $\kappa$, and that all these values can be fixed simultaneously for all regular $\lambda$. Reasonable here means that $2^{\lambda}$ is limited only by the usual restrictions on such values and $\kappa$ is as above. The other states that the strong complexity for $G(\lambda, \kappa)$ can be any regular cardinal between $\lambda^{+}$and $2^{\lambda}$ for reasonable 
values of $2^{\lambda}$ and $\kappa$, and that these values can all be fixed simultaneously for all regular $\lambda$. These results will be stated in the sections 2 and 3 , thus, the paper can be considered self-contained.

In sections 2 and 3, we take the global product forcings given in [3] and modify them so that they are reverse Easton iterations. In both cases we are fixing the value of $F(\lambda)=2^{\lambda}$ for all regular $\lambda$ simultaneously. This could lead to problems as the values that $F$ takes can skip over cardinals. However, there is a club class of cardinals $\lambda$ such that $F(\theta)<\lambda$ for all $\theta<\lambda$. We will form our iteration over these closure points. This is important in order to build generics.

In sections 4 and 5, we build generics for the global iterations given in Sections 2 and 3.

In section 6, we isolate the special properties of our forcings which are required for our construction. As our forcings lack the homogeneity properties of Cohen forcing which were heavily used in [7], we instead rely on other distinctive properties of the forcing in order to directly build the generic. We also discuss other types of forcings which may meet these criteria.

We will use the convention that for $p, q \in P$, a forcing notion, $q \leq p$ indicates that $q$ is stronger than $p$. For a forcing condition $p$, we denote by $\operatorname{Dom}(p)$ the non-trivial domain, or support, of $p$.

\section{Iterating high complexity}

In the paper [3] a forcing notion was given for any regular uncountable $\lambda$ which fixed the complexity of $\mathcal{C}(\lambda, \kappa(\lambda))$ to be maximal (that is, $2^{\lambda}$ ) and fixed the value of $2^{\lambda}>\lambda^{+}$. A globalisation of that result was also given as an Easton product, which proved the following result:

Theorem 2.1. Assume $V=L$. To each uncountable regular cardinal $\lambda$ associate a regular cardinal $\kappa(\lambda) \leq \lambda$ and a cardinal $F(\lambda)$ such that $\operatorname{cf}(F(\lambda))>\lambda$ and $\lambda<\theta$ implies $F(\lambda) \leq F(\theta)$. Assume that the functions $\kappa, F$ are $L$ definable. Then there exists an $L$-definable, ZFC-preserving, cardinal preserving class forcing notion $P$ such that in $L^{P}$ the complexity of $\mathcal{C}(\lambda, \kappa(\lambda))$ 
is $F(\lambda)=2^{\lambda}$ for each regular uncountable $\lambda$.

Remark 2.2. In the theorem above, the functions $\kappa$ and $F$ are $L$-definable with or without parameters. When building the generic in Section 4, it will be required that these functions are definable without parameters.

Namely, $P$ was defined as a product forcing notion of $P(\lambda, \kappa(\lambda), F(\lambda)$ ) (defined below) with Easton support. Recall that Easton support means that the support is bounded at inaccessibles.

The forcing notion $P(\lambda, \kappa(\lambda), F(\lambda))$ was defined to be the $(<\lambda)$-support product of $F(\lambda)$ copies of $Q=Q(\lambda, \kappa(\lambda))$. We will repeat the definition of $Q$ below, but first a remark is needed about the terminology.

The definition of $Q$ requires a set of bounded subsets of $\lambda$ which we call low sets. These are used to restrict the growth of chains when extending sequences of posets without creating large antichains in the forcing. This method was introduced in [9] and also used in [3]. The exact definition is not required for this paper, but can be found in both of the papers above.

Let the elements of $Q$ be $q=(\delta, X, \mathcal{A})$, where

1. $\delta<\lambda$ is an ordinal,

2. $X \subseteq \delta$ is a poset $\left(X, \leq_{X}\right)$ which omits $\kappa(\lambda)$-chains and in which $\alpha \leq_{X} \beta$ implies that $\alpha \leq \beta$ (as ordinals),

3. the conditions on $\mathcal{A}$ are as follows:

(a) $\mathcal{A}$ is a family of size $<\lambda$ of low subsets $A$ of $\lambda$ such that $A \subseteq \delta$ and $|A|<\kappa(\lambda)$,

(b) if $A \in \mathcal{A}$ is such that $\sup (A) \leq x<\delta$, then $A \leq_{X} x$.

The ordering of $Q$ is as follows. If $q=(\delta, X, \mathcal{A})$ and $q^{\prime}=\left(\delta^{\prime}, X^{\prime}, \mathcal{A}^{\prime}\right)$ are in $Q$, then $q^{\prime} \leq_{Q} q$ (i.e. $q^{\prime}$ is stronger than $q$ ) if and only if $\delta^{\prime} \geq \delta$, with $X$ a subposet of $X^{\prime}$ such that $X=X^{\prime} \cap \delta$ and $\mathcal{A}=\mathcal{A}^{\prime} \cap[\delta]^{<\kappa(\lambda)}$.

If a forcing notion has the property that one can build decreasing sequences of size $<\lambda$ only controlling what happens at limit stages, we say that the forcing is weakly $\lambda$-closed. More precisely: 
Definition 2.3. A forcing notion $P$ is weakly $\lambda$-closed, if and only if there exists a function $w: P^{<\lambda} \rightarrow P$ such that for all limit $\tau<\lambda$ if $\left\langle p_{\alpha}: \alpha<\tau\right\rangle$ is a descending chain and satisfies the following:

$$
\text { for all limit } \tau^{\prime}<\tau \text { we have } p_{\tau^{\prime}} \leq w\left(\left\langle p_{\alpha}: \alpha<\tau^{\prime}\right\rangle\right)
$$

then the sequence $\left\langle p_{\alpha}: \alpha<\tau\right\rangle$ has a lower bound.

Note that weakly $\lambda$-closed falls in between the notions of $\lambda$-closed and $\lambda$ strategically closed. The forcing $P(\lambda, \kappa(\lambda), F(\lambda))$ was shown to be weakly $\lambda$-closed and satisfy the $\lambda^{+}$-cc.

In order to build a generic for the global result Theorem 2.1, we must first have an iteration.

Lemma 2.4. We can find a class forcing $P$ such that it satisfies the conditions of Theorem 2.1 and in addition, $P$ is given as a reverse Easton iteration $\left\langle\left(P_{\alpha},{\underset{\sim}{\alpha}}_{\alpha}\right): \alpha \in \operatorname{Ord}\right\rangle$ where $P_{\alpha} \Vdash{\underset{\sim}{\alpha}}_{\alpha}$ is weakly $\alpha$-closed for each $\alpha \in$ Ord.

Proof To form an iteration out of this, we must iterate over the closure points of $F$ and use a product in between.

Let $\left\langle\lambda_{\beta}: \beta \in\right.$ Ord $\rangle$ be the class of closure points of the the $F$ function. That is, for all ordinals $\beta$ and for all $\theta<\lambda_{\beta}$, a regular cardinal, $F(\theta)<\lambda_{\beta}$. Note that each $\lambda_{\beta}$ is a limit cardinal.

Define $\left\langle\left(P_{\alpha}, Q_{\alpha}\right): \alpha \in \operatorname{Ord}\right\rangle$ as follows. Let $Q_{\alpha}$ be the Easton support product of $P(\beta, \kappa \tilde{(} \beta), F(\beta))$ for $\beta \in\left[\lambda_{\alpha}, \lambda_{\alpha+1}\right)$ a regular, uncountable cardinal. Let $P$ be the reverse Easton iteration of the $Q_{\alpha}$.

As in [3], $Q_{\alpha}$ preserves cofinalities and forces that $\mathcal{C}(\beta, \kappa(\beta))$ has complexity $F(\beta)$ for all $\beta \in\left[\lambda_{\alpha}, \lambda_{\alpha+1}\right)$.

We must only check that at closure points $\lambda_{\beta}$, the complexity has not been affected by the forcing below $\lambda_{\beta}$. Since $\lambda_{\beta}$ is always a limit cardinal, we must check that the complexity of $\mathcal{C}(\lambda, \kappa(\lambda))$ is $F(\lambda)$ in $M^{P_{\lambda}}$ if $\lambda=\lambda_{\beta}$ is inaccessible or $\lambda=\lambda_{\beta}^{+}$and $\lambda_{\beta}$ is singular.

When $\lambda_{\beta}$ is inaccessible, we will have two cases, depending on whether or not it is Mahlo. If $\lambda_{\beta}$ is a Mahlo cardinal, then the forcing has the $\lambda_{\beta}$-cc and the result follows as in [3]. 
So assume that $\lambda_{\beta}$ is not Mahlo. We will use the elementary submodels argument as in [3] to check that we have the correct complexity. In this case, we only have the $\lambda_{\beta}^{+}$-cc. We must ensure that the forcing below $\lambda_{\beta}$ does not introduce inconvenient embeddings which would lower the complexity at $\lambda_{\beta}$. To that end, we can anticipate a generic for the forcing below $\lambda_{\beta}$ and run the complexity argument based on the information in the anticipated generic. As we have the $\lambda_{\beta}^{+}$-cc, we can anticipate a maximal antichain in $\lambda_{\beta}$ steps and we use elementary submodels to ensure that this happens in order type $\lambda_{\beta}$.

If $\lambda_{\beta}$ is singular, then the forcing at $\lambda_{\beta}$ is trivial. However, we must check the complexity at $\lambda_{\beta}^{+}$. Here we have the $\lambda_{\beta}^{++}$-cc. The argument then proceeds as in the non-Mahlo inaccessible case, replacing $\lambda_{\beta}$ with $\lambda_{\beta}^{+}$.

\section{Iterating low complexity}

We will also make an iteration out of the globalisation of the low complexity result in [9]. As before, we have already proved in [3] that the forcing below exists as a product.

Theorem 3.1. Assume $V=L$. Let $\lambda, \kappa(\lambda), F$ be as in Theorem 2.1 and let $\nu(\lambda) \in\left[\lambda^{+}, F(\lambda)\right)$ be $L$-definable. Then there exists an $L$-definable, ZFCpreserving, cardinal preserving class forcing notion $P$ such that in $L^{P}$ the strong complexity of $G(\lambda, \kappa(\lambda))$ is $\nu(\lambda)$ and $F(\lambda)=2^{\lambda}$ for each regular uncountable $\lambda$.

Remark 3.2. In the theorem above, the functions $\kappa, \nu$ and $F$ are $L$-definable with or without parameters. When building the generic in Section 4, it will be required that these functions are definable without parameters.

The general construction of this global iteration is as before, except at each regular cardinal $\lambda$, the forcing is an iteration of length $\nu(\lambda)$ which produces a member of a universal family at every successor stage. When necessary in order to distinguish the local iteration at a particular $\lambda$ and the global iteration, the local iteration will have a superscript $l$.

Here, the global forcing $P$ will be defined as an iterated forcing notion with Easton support. At each regular stage $\lambda$ of this global iteration, we force with 
$P(\lambda, F(\lambda), \kappa(\lambda), \nu(\lambda))=\left\langle P_{\alpha}^{l}, Q_{\alpha}^{l}: 0 \leq \alpha<\nu(\lambda)\right\rangle$ which is a $(<\lambda)$-support iteration of length $\nu(\lambda)$. At singular stages, the forcing will be trivial. For a particular regular $\lambda$, we describe the forcing $P(\lambda, F(\lambda), \kappa(\lambda), \nu(\lambda))$ below.

The first step of the iteration, $Q_{0}^{l}$, is used to add $F(\lambda)$-many Cohen subsets to $\lambda$, using the standard $\lambda^{+}$-cc and $\lambda$-closed forcing.

In each step $\alpha \geq 1$ of the iteration, we will add a graph of size $\lambda$ which omits $\kappa(\lambda)$-cliques that strongly embeds every member of $G(\lambda, \kappa(\lambda))$ in $M^{P_{\alpha}^{l}}$. We will modify the conditions for $Q_{\alpha}^{l}$ slightly from the version in [9] in order to make the construction of the generic easier.

Namely, let $Q_{\alpha}^{l}=(Q, \leq)$ for some ordinal $\alpha$ such that $1 \leq \alpha<\nu(\lambda)$. Fix some "canonical" enumeration $\left\{Z_{\gamma}: \gamma<F(\lambda)\right\}$ of all graphs of size $\lambda$ which omit $\kappa(\lambda)$-cliques which exist at this stage. Let $q \in Q$ if and only if $q=(\delta, X, \mathcal{A}, \mathcal{Z}, \Phi)$ with the following properties:

1. $\delta<\lambda$ is an ordinal,

2. $X \subseteq \delta$ is a graph which omits $\kappa(\lambda)$-cliques,

3. $\mathcal{A} \subseteq[\delta]^{<\kappa(\lambda)}$ is a family of low subsets of $\delta$ such that $|\mathcal{A}|<\lambda$,

4. $\mathcal{Z} \subseteq F(\lambda)$ such that $|\mathcal{Z}|<\lambda$,

5. $\Phi: \mathcal{Z} \times \delta \rightarrow \delta$ is a function such that if $\gamma \in \mathcal{Z}$, then the mapping $x \mapsto \Phi(\gamma, x)$ is a strong embedding from $Z_{\gamma} \uparrow \delta$ into $X$,

6. if $A \in \mathcal{A}$ is such that $\sup (A) \leq x<\delta$, then $A \times\{x\} \nsubseteq X$ (i.e. there exists an element of $A$ which is not connected to $x$ in $X$ ),

7. if $A \in \mathcal{A}$ and $\gamma \in \mathcal{Z}$, then $A \nsubseteq \Phi ”\left(\left\{Z_{\gamma}\right\} \times \delta\right):=\left\{\Phi\left(Z_{\gamma}, \beta\right): \beta<\delta\right\}$.

If $q=(\delta, X, \mathcal{A}, \mathcal{Z}, \Phi)$ and $q^{\prime}=\left(\delta^{\prime}, X^{\prime}, \mathcal{A}^{\prime}, \mathcal{Z}^{\prime}, \Phi^{\prime}\right)$ are in $Q$, then $q^{\prime} \leq_{Q} q$ (i.e. $q^{\prime}$ is stronger than $q$ ) if $\delta^{\prime} \geq \delta$, with $X=X^{\prime} \cap \delta$ as graphs, $\mathcal{A}=\mathcal{A}^{\prime} \cap[\delta]^{<\kappa(\lambda)}$ and $\mathcal{Z} \subseteq \mathcal{Z}^{\prime}$. Additionally, the following requirements must be met:

(a) if $\left(Z_{\gamma}, x\right) \in \operatorname{Dom}(\Phi)$ then $\Phi^{\prime}\left(Z_{\gamma}, x\right)=\Phi\left(Z_{\gamma}, x\right)$,

(b) if $\gamma \in \mathcal{Z}$ and $\delta \leq x<\delta^{\prime}$ then $\Phi^{\prime}\left(Z_{\gamma}, x\right) \geq \delta$, 
(c) if $\gamma \neq \gamma^{\prime} \in \mathcal{Z}$ and $\delta \leq x, y<\delta^{\prime}$, then $\Phi^{\prime}\left(Z_{\gamma}, x\right) \neq \Phi^{\prime}\left(Z_{\gamma^{\prime}}, y\right)$.

The intuition for the definition of conditionhood is as follows. The graph $X$ generically becomes a model which is universal for graphs in $G(\lambda, \kappa(\lambda))$ which exist at that stage of the iteration. The set $\mathcal{A}$ prevents large cliques from forming in $X$ via condition 6 . The graphs indexed by $\mathcal{Z}$ are the "ground model" graphs which exist at that stage of the iteration whose restriction to $\delta$ embed into $X$. The functions $\Phi$ are the appropriate partial embeddings.

The modification that we made to the conditions was to take the actual graphs of size $\lambda$ out of the condition and replace them by their indices. This changes the effective size of the conditions to be $<\lambda$.

Forming the global iteration for the forcing $P$ works exactly as in Section 2. Namely, let $\left\langle\lambda_{\beta}: \beta \in\right.$ Ord $\rangle$ be the class of closure points of the the $F$ function as before. Define $P=\left\langle\left(P_{\alpha}, Q_{\alpha}\right): \alpha \in\right.$ Ord $\rangle$ as follows. Let $Q_{\alpha}$ be the Easton support product of $P(\beta, \tilde{\kappa(\beta)}, F(\beta), \nu(\beta))$ for $\beta \in\left[\lambda_{\alpha}, \lambda_{\alpha+1}\right)$ a regular, uncountable cardinal. Let $P$ be the reverse Easton iteration of the $Q_{\alpha}$. Using similar techniques as in the case of high complexity, $P$ forces the complexity of $G(\lambda, \kappa(\lambda))$ to be $\nu(\lambda)$ for all regular $\lambda$.

\section{Generic for high complexity}

In this section, we will build a generic for the forcing $P$ such that in $M^{P}$ the complexity of $\mathcal{C}(\lambda, \kappa(\lambda))$ is $F(\lambda)=2^{\lambda}$ for all regular $\lambda$. Namely, this is the forcing $P$ as defined in Lemma 2.4 above.

Let $I=\left\{i_{\alpha}: \alpha \in\right.$ Ord $\}$ be the Silver indiscernibles for $L$ in increasing order. We will make use of the following facts:

1. For all formulas $\varphi$, it is the case that $L \vDash \varphi(\alpha, \vec{i}) \Leftrightarrow \varphi(\alpha, \vec{j})$ whenever $\alpha<\min (\vec{i} \cup \vec{j})$ where $\vec{i}, \vec{j} \in I^{<\omega}$.

2. If $t$ is a Skolem term in $L$ and $t\left(j_{1}, \ldots, j_{k}, j_{k+1}, \ldots, j_{n}\right)<j_{k+1}$ where $j_{1}<\ldots<j_{k}<j_{k+1}<\ldots<j_{n}$, then this term has the same value as $t\left(j_{1}, \ldots, j_{k}, j_{k+1}^{\prime}, \ldots, j_{n}^{\prime}\right)$ where $j_{i}^{\prime}>j_{k}$ for $i>k$. 
Since the second fact indicates that the parameters above $j_{k}$ are irrelevant (when $t$ takes values below $\left.j_{k+1}\right)$, we often write $t\left(j_{1}, \ldots, j_{k}, \vec{\infty}\right)$. When we say that $\vec{j}<\alpha$ for some vector $j$, we mean that all elements of $\vec{j}$ are less than $\alpha$.

There are $\aleph_{0}$ many Skolem terms, so we will enumerate them as $\left\{t_{n}: n<\omega\right\}$.

Theorem $4.1\left(0^{\#}\right)$. Let $P_{\infty}=\langle P(\alpha): \alpha \in \operatorname{Ord}\rangle$ be the iteration with Easton support definable in $L$ without parameters as in Lemma 2.4 above. Also assume that the functions $\kappa$ and $F$ (defined in Theorem 2.1) are $L$-definable without parameters. Then there exists a $G$ such that $G$ is $P_{\infty}$-generic over $L$.

Proof First note that it suffices to meet all set maximal antichains since there are no cofinal antichains in $P_{\infty}$. We will define $G(\leq i)$ generic for $P(\leq i)$ by induction on $i \in I$. Since the function $F$ is definable without parameters, the indiscernibles are closure points of $F$.

The following properties of $P$ were proved in the previous paper.

Lemma 4.2. $\quad$ 1. $P(>\lambda)$ is weakly $\lambda^{+}$-closed for all regular $\lambda$.

2. $P(<\lambda)$ has the $\lambda$-cc for Mahlo (and hence, indiscernible) $\lambda$.

Finding a generic for $P\left(\leq i_{0}\right)$ is trivial since $\mathcal{P}\left(P\left(\leq i_{0}\right)\right)^{L}$ is countable.

If $\alpha$ is a limit ordinal then we want $G\left(\leq i_{\alpha}\right)$ to be the "direct limit" of $G\left(\leq i_{\beta}\right)$ for $\beta<\alpha$. In order to achieve the compatibility needed to make this generic, we will use the shift map below.

Let $\beta<\beta^{\prime}$. We will define $\pi_{i_{\beta}, i_{\beta^{\prime}}}$ as follows:

$$
\pi_{i_{\beta}, i_{\beta^{\prime}}}\left(i_{\gamma}\right)= \begin{cases}i_{\gamma} & \gamma<\beta \\ i_{\beta^{\prime}+(\gamma-\beta)} & \gamma \geq \beta\end{cases}
$$

Shifting one indiscernible up to another extends uniquely to an elementary embedding from $L$ into $L$. We will abuse notation and denote this extension in the same way. 
Let $G\left(\leq i_{\beta}\right) \rightarrow G\left(\leq i_{\beta^{\prime}}\right)$ denote that $G\left(\leq i_{\beta}\right)$ embeds into $G\left(\leq i_{\beta^{\prime}}\right)$ in the sense that $\pi_{i_{\beta}, i_{\beta^{\prime}}}\left[G\left(\leq i_{\beta}\right)\right] \subseteq G\left(\leq i_{\beta^{\prime}}\right)$. Using the shift map we want to find $G\left(\leq i_{\beta^{\prime}}\right)$ such that $G\left(\leq i_{\beta}\right) \rightarrow G\left(\leq i_{\beta^{\prime}}\right)$ and $G\left(i_{\beta^{\prime}}\right)$ is generic for $P\left(i_{\beta^{\prime}}\right)$.

Lemma 4.3. If $G\left(\leq i_{\beta}\right) \rightarrow G\left(\leq i_{\beta^{\prime}}\right)$ for all $\beta<\beta^{\prime}<\alpha$ for $\alpha$ limit, then the direct limit $G\left(\leq i_{\alpha}\right)=\bigcup_{\beta<\alpha} \pi_{i_{\beta}, i_{\alpha}}\left[G\left(\leq i_{\beta}\right)\right]$ is generic for $P\left(\leq i_{\alpha}\right)$.

Proof Let $\Delta=t\left(\vec{i}, i_{\alpha}, \vec{\infty}\right)$ be a maximal antichain in $P\left(\leq i_{\alpha}\right)$. Then $\bar{\Delta}=$ $t\left(\vec{i}, i_{\beta}, \vec{\infty}\right)$ is a maximal antichain in $P\left(\leq i_{\beta}\right)$. If $p \in G\left(\leq i_{\beta}\right) \cap \bar{\Delta}$ and $G\left(\leq i_{\beta}\right) \rightarrow G\left(\leq i_{\alpha}\right)$ then $\pi_{i_{\beta}, i_{\alpha}}(p) \in \Delta \cap G\left(\leq i_{\alpha}\right)$.

The successor case, $i_{\alpha+1}$ is the interesting one. We wish to find $G\left(\leq i_{\alpha+1}\right)$ assuming that we have already built $G\left(\leq i_{\alpha}\right)$. We first note that $P\left(<i_{\alpha+1}\right)=$ $P\left(\leq i_{\alpha}\right) * P\left(i_{\alpha}, i_{\alpha+1}\right)$ where $P\left(i_{\alpha}, i_{\alpha+1}\right)$ is the forcing in the interval $\left(i_{\alpha}, i_{\alpha+1}\right)$. By the induction hypothesis, we have $G\left(\leq i_{\alpha}\right)$ which is $P\left(\leq i_{\alpha}\right)$-generic. We can split our task up into finding $G\left(i_{\alpha}, i_{\alpha+1}\right)$, generic for $P\left(i_{\alpha}, i_{\alpha+1}\right)$ and then finding $G\left(i_{\alpha+1}\right)$ which is $P\left(i_{\alpha+1}\right)$-generic.

We start by building $G\left(i_{\alpha}, i_{\alpha+1}\right)$. First note that by Lemma $4.2(2)$, every antichain in $P\left(i_{\alpha}, i_{\alpha+1}\right)$ has size $<i_{\alpha+1}$. We will show how to group all open dense subsets of $P\left(i_{\alpha}, i_{\alpha+1}\right)$ in $L_{i_{\alpha+1}}\left[G\left(\leq i_{\alpha}\right)\right]$ into $\aleph_{0}$ many families each of size $i_{\alpha}$. Then we can use the weak $i_{\alpha}^{+}$-closure of $P\left(i_{\alpha}, i_{\alpha+1}\right)$ to handle maximal antichains in each family.

Lemma 4.4. Let $D$ be the collection of all open dense sets in $P\left(i_{\alpha}, i_{\alpha+1}\right)$ which belong to $L_{i_{\alpha+1}}\left[G\left(\leq i_{\alpha}\right)\right]$. Then $D=\bigcup_{n<\omega} D_{n}$ such that each $D_{n} \in$ $L_{i_{\alpha+1}}\left[G\left(\leq i_{\alpha}\right)\right]$ and $\left|D_{n}\right|=i_{\alpha}$.

Proof For every $x \in L_{i_{\alpha+1}}\left[G\left(\leq i_{\alpha}\right)\right]$, there exists an $n$ such that $x=t_{n}(\vec{j}, \vec{k})$ for $\vec{j}<i_{\alpha}$ and $\vec{k} \geq i_{\alpha+1}$. As we are only considering subsets of $i_{\alpha+1}$, we know that $\vec{k}$ is irrelevant to the value of $t_{n}$. We will arrange all elements of $L_{i_{\alpha+1}}\left[G\left(\leq i_{\alpha}\right)\right]$ according to the enumeration of the Skolem terms for which they take values.

Let $D_{n}$ be the set of all open dense subsets $t_{n}^{L\left[G\left(\leq i_{\alpha}\right)\right]}(\vec{\beta}, \vec{\infty})$ of $P\left(i_{\alpha}, i_{\alpha+1}\right)$ such that $\vec{\beta} \leq i_{\alpha}$ is a finite set of ordinals, $t_{n}^{L\left[G\left(\leq i_{\alpha}\right)\right]}(\vec{\beta}, \vec{\infty}) \in L_{i_{\alpha+1}}\left[G\left(\leq i_{\alpha}\right)\right]$ and $\vec{\infty}$ is a finite set of fixed indiscernibles of the appropriate length. $L$ cannot see indiscernibles, so we take all ordinal vectors instead which will include the indiscernibles. 
So, for each $n, D_{n} \in L\left[G\left(\leq i_{\alpha}\right)\right]$. Now $\left|D_{n}\right|^{L\left[G\left(i_{\alpha}\right)\right]}=i_{\alpha}$ and there are only countably many of them.

Thus, the sequence of the $D_{n}$ 's is not in $L\left[G\left(\leq i_{\alpha}\right)\right]$, but the individual pieces are. We can also show that the shift of a $D_{n}$ will also be in $L\left[G\left(\leq i_{\alpha}\right)\right]$.

Claim 4.5. 1. If $X \in L$ and $|X| \leq i_{\beta^{\prime}}$ then $\operatorname{Ran}\left(\pi_{i_{\beta}, i_{\beta^{\prime}}}\right) \cap X \in L$.

2. If $X \in L$ and $|X| \leq i_{\beta^{\prime}}$ then $\pi_{i_{\beta}, i_{\beta^{\prime}}} \uparrow X \in L$.

Proof The proof of (1) breaks down into two cases. In the first case, we have $X \in \operatorname{Ran}\left(\pi_{i_{\beta}, i_{\beta^{\prime}}}\right)$. Let $s: X \rightarrow i_{\beta^{\prime}}$ be a bijection which is in $L$ such that $s \in \operatorname{Ran}\left(\pi_{i_{\beta}, i_{\beta^{\prime}}}\right)$. Therefore, $\operatorname{Ran}\left(\pi_{i_{\beta}, i_{\beta^{\prime}}}\right) \cap X=s^{-1}\left[\operatorname{Ran}\left(\pi_{i_{\beta}, i_{\beta^{\prime}}}\right) \cap i_{\beta^{\prime}}\right]$. Since the critical point of $\pi_{i_{\beta}, i_{\beta^{\prime}}}$ is $i_{\beta}$, this is equal to $s^{-1}\left[i_{\beta}\right]$, which is in $L$.

If $X \notin \operatorname{Ran}\left(\pi_{i_{\beta}, i_{\beta^{\prime}}}\right)$ we can show that there exists $Y \in L$ with $Y \in \operatorname{Ran}\left(\pi_{i_{\beta}, i_{\beta^{\prime}}}\right)$ and $X \subseteq Y$.

First note that $\operatorname{Ran}\left(\pi_{i_{\beta}, i_{\beta^{\prime}}}\right)=\left\{t\left(\vec{\alpha}, i_{\beta^{\prime}}, \vec{k}\right): \vec{\alpha}<i_{\beta}, \vec{k}>i_{\beta^{\prime}}\right\}$. Also there is some Skolem term $t$ such that $X=t\left(\vec{\gamma}, i_{\beta}, i_{\beta^{\prime}}, \vec{l}\right)$ for some $\vec{\gamma}<i_{\beta}$ and $\vec{l}>i_{\beta^{\prime}}$. Let

$$
\begin{gathered}
Y=\bigcup\left\{t\left(\vec{\alpha}, \delta, i_{\beta^{\prime}}, \vec{j}\right): \delta \in\left(\max (\vec{\alpha}), i_{\beta^{\prime}}\right), \delta \in \operatorname{Ord},\right. \\
\left.\left|t\left(\vec{\alpha}, \delta, i_{\beta^{\prime}}, \vec{j}\right)\right|=i_{\beta^{\prime}}\right\}
\end{gathered}
$$

One can see that $Y \in L$ as all parameters are either ordinals or fixed. It is also the case that $Y \supseteq \operatorname{Ran}\left(\pi_{i_{\beta}, i_{\beta^{\prime}}}\right) \cap X$. Thus,

$$
\operatorname{Ran}\left(\pi_{i_{\beta}, i_{\beta^{\prime}}}\right) \cap X=\left(\operatorname{Ran}\left(\pi_{i_{\beta}, i_{\beta^{\prime}}}\right) \cap Y\right) \cap X \in L .
$$

To prove (2), let $f: i_{\beta^{\prime}} \rightarrow X$ be a bijection in $L$. For all $x \in X$, we have

$$
\begin{aligned}
\pi_{i_{\beta}, i_{\beta^{\prime}}}(x) & =\pi_{i_{\beta}, i_{\beta^{\prime}}}\left(f\left(f^{-1}(x)\right)\right) \\
& =\pi_{i_{\beta}, i_{\beta^{\prime}}}(f) \pi_{i_{\beta}, i_{\beta^{\prime}}}\left(f^{-1}(x)\right) \\
& =\pi_{i_{\beta}, i_{\beta^{\prime}}}(f)\left(f^{-1}(x)\right) .
\end{aligned}
$$


The conclusion is clear as $f$ and $f^{-1}(x)$ are in $L$.

We will build $G\left(i_{\alpha}, i_{\alpha+1}\right)$ by induction on $n$ in the enumeration of the Skolem terms. By the weak $i_{\alpha}^{+}$-closure of $P\left(i_{\alpha}, i_{\alpha+1}\right)$ we can find $p_{0} \in P\left(i_{\alpha}, i_{\alpha+1}\right)$ such that $p_{0}$ meets every dense set in $D_{0}$. This can be done since there can only be $i_{\alpha}$ many of them. Assume that we have found $\left\langle p_{m}: m<n\right\rangle$ such that $p_{m}$ is compatible with all $p_{m^{\prime}}$ where $m^{\prime}<m$ and meets all dense sets in $D_{m}$. Use the weak closure again to find $p_{n}$ such that $p_{n}$ is compatible with all $p_{m}$ where $m<n$ and meets all dense sets in $D_{n}$. Let $G\left(i_{\alpha}, i_{\alpha+1}\right)=\left\{p_{n}: n<\omega\right\}$.

Now we must find $G\left(i_{\alpha+1}\right)$, a generic for $P\left(i_{\alpha+1}\right)$. We do this by first shifting the conditions in $G\left(i_{\alpha}\right)$ up to $i_{\alpha+1}$.

We will show that if we shift the conditions in the generic up to $i_{\alpha+1}$, they remain conditions in $P_{i_{\alpha+1}}$. So we need to see that each poset that we build generically at $i_{\alpha}$ omits $\kappa\left(i_{\alpha+1}\right)$-chains. Because the forcing is $L$-definable without parameters, $\kappa\left(i_{\alpha}\right)$ is either $i_{\alpha}$ or some $\theta<i_{0}$, the least indiscernible. In both cases, we have $\kappa\left(i_{\alpha}\right) \leq \kappa\left(i_{\alpha+1}\right)$. Consider $\pi_{i_{\alpha}, i_{\alpha+1}}\left[G\left(i_{\alpha}\right)\right]$. This is a set of posets of size $i_{\alpha}$ which omit chains of size $\pi_{i_{\alpha}, i_{\alpha+1}}\left(\kappa\left(i_{\alpha}\right)\right)=\kappa\left(i_{\alpha+1}\right)$.

Let $\pi_{i_{\alpha}, i_{\alpha+1}}\left[G\left(i_{\alpha}\right)\right]=\left\{p_{\beta}: \beta<F\left(i_{\alpha}\right)\right\}$. Since we are taking the pointwise image of the generic at $i_{\alpha}$ and the conditions have size $<i_{\alpha}$, this remains the case in the image. Our goal is to extend this image to a generic for $P\left(i_{\alpha+1}\right)$.

The idea is to break up the product at $i_{\alpha}$ into $\omega$-many pieces each of size $i_{\alpha}$, call them $M_{n}^{\alpha}$ for $n<\omega$. The $M_{n}^{\alpha}$ 's will be increasing, that is, $M_{n}^{\alpha} \subseteq M_{n+1}^{\alpha}$. The sequence of pieces is not in $L\left[G\left(\leq i_{\alpha}\right)\right]$, but each piece is. For each $n$ we will form the image of the generic $G\left(i_{\alpha}\right)$ restricted to $M_{n}^{\alpha}$ and then extend this to form a generic for the corresponding piece of the forcing $P\left(i_{\alpha+1}\right)$.

For each $\alpha \in$ Ord and $n<\omega$ let $M_{n}^{\alpha}$ be the $\Sigma_{1} \operatorname{Hull}\left(i_{\alpha} \cup\left\{i_{\alpha}, i_{\alpha+1}, \ldots, i_{\alpha+n}\right\}\right)$. These are $\Sigma_{1}$-elementary submodels of $L$ and are $i_{\alpha}$-closed.

For $n=1$, let $\left\{p: p \in M_{1}^{\alpha} \cap G\left(i_{\alpha}\right)\right\}=: A_{1}$. Then $\pi_{i_{\alpha}, i_{\alpha+1}}\left[A_{1}\right]$ is a set of conditions of $P\left(i_{\alpha+1}\right)$ and there are only $i_{\alpha}$-many of them.

Let $p_{1}^{*}=\bigcup \pi_{i_{\alpha}, i_{\alpha+1}}\left[A_{1}\right]$. To see that this is a condition, we must show that for each component in the support, no $\kappa_{\alpha+1}$ sequences have been added. One may write $p_{1}^{*}=\bigcup\left\{\pi_{i_{\alpha}, i_{\alpha+1}}(\langle p(\beta): \beta \in \operatorname{Dom}(p)\rangle): p \in A_{1}\right\}$ which means that $p_{1}^{*}(\beta)=\bigcup\left\{\pi_{i_{\alpha}, i_{\alpha+1}}(p)(\beta): p \in A_{1}\right\}$ for each $\beta \in \operatorname{Dom}\left(p_{1}^{*}\right)$. We need to see 
that $X_{p_{1}^{*}(\beta)}$ omits $\kappa\left(i_{\alpha+1}\right)$-chains. This has been proved by [3, Lemma 3.9], where it was shown that the generic poset $\bigcup\left\{X:(\delta, X, \mathcal{A}) \in G_{Q}\right.$ generic for $\left.Q\left(i_{\alpha}, \kappa\left(i_{\alpha}\right)\right)\right\}$ (using the notation for the forcing from Section 2) has no $\kappa\left(i_{\alpha}\right)$-chain.

The filtration $\left\{M_{n}^{\alpha}: n<\omega\right\}$ can be shifted up to a filtration of $P\left(i_{\alpha+1}\right)$, namely $M_{n}^{\alpha+1}=\pi_{i_{\alpha}, i_{\alpha+1}}\left(M_{n}^{\alpha}\right)$. Note that each of the $M_{n}^{\alpha+1}$ has size $i_{\alpha+1}$, but there are still only $\omega$-many of them. Thus, if $i_{\alpha}$ is a limit indiscernible, $M_{1}^{\alpha}$ is the direct limit of $\pi_{i_{\beta}, i_{\alpha}}\left(M_{1}^{\beta}\right)$ for $\beta<\alpha$.

Since $M_{1}^{\alpha+1}$ is $i_{\alpha+1}^{+}$-closed and $p_{1}^{*}$ is a lower bound for $\pi_{i_{\alpha}, i_{\alpha+1}}\left[A_{1}\right]$, we have $p_{1}^{*} \in M_{1}^{\alpha+1}$. We may extend below $p_{1}^{*}$ to form a generic for $M_{1}^{\alpha+1}$ by breaking up the dense sets that we must meet into $\omega$-many blocks as before. We call this restricted generic $G^{1}\left(i_{\alpha+1}\right)$.

The case $n=2$ is essentially the general case. Again form $A_{2}:=\left\{p_{\beta} \in\right.$ $\left.G\left(i_{\alpha}\right) \cap M_{2}^{\alpha}: \beta<i_{\alpha}\right\}$. Extend below $\pi_{i_{\alpha}, i_{\alpha+1}}\left[A_{2}\right]$ to form $p_{2}^{*} \in M_{2}^{\alpha+1}$. Here we need to check that $p_{2}^{*}$ is compatible with $G^{1}\left(i_{\alpha+1}\right)$.

One can decompose $p_{2}^{*}=p_{2}^{*} \uparrow M_{1}^{\alpha+1} \cup p_{2}^{*} \uparrow\left(M_{2}^{\alpha+1}-M_{1}^{\alpha+1}\right)$. Note that $p_{2}^{*} \uparrow M_{1}^{\alpha+1} \in M_{1}^{\alpha+1}$ by the $i_{\alpha+1}$-closure of $M_{1}^{\alpha+1}$. If there were a compatibility issue with $p_{2}^{*} \uparrow M_{1}^{\alpha+1}=p_{1}^{*} \geq G^{1}\left(i_{\alpha+1}\right)$, then this would have already been a problem in $M_{1}^{\alpha+1}$ which would contradict the genericity of $G^{1}\left(i_{\alpha+1}\right)$. However, $p_{2}^{*}\left\lceil\left(M_{2}^{\alpha+1}-M_{1}^{\alpha+1}\right)\right.$ is irrelevant to $G^{1}\left(i_{\alpha+1}\right)$, that is, the conditions of $G^{1}\left(i_{\alpha+1}\right)$ are not defined in this domain.

Now, as $p_{2}^{*}$ is compatible with $G^{1}\left(i_{\alpha+1}\right)$, we may extend below $p_{2}^{*}$ to form a generic $G^{2}\left(i_{\alpha+1}\right)$ for $M_{2}^{\alpha+1}$ such that $G^{1}\left(i_{\alpha+1}\right) \subseteq G^{2}\left(i_{\alpha+1}\right)$.

Continue this process for all $n<\omega$. Let $G\left(i_{\alpha+1}\right)=\bigcup_{n<\omega} G^{n}\left(i_{\alpha+1}\right)$. We would like to show that $G\left(i_{\alpha+1}\right)$ is generic for $P\left(i_{\alpha+1}\right)$. Recall that $P\left(i_{\alpha+1}\right)$ has the $i_{\alpha+1}^{+}$-cc so any antichain has size at most $i_{\alpha+1}$. Any maximal antichain must be contained in $M_{n}^{\alpha+1}$ for some $n<\omega$, since each $M_{n}^{\alpha+1}$ is $i_{\alpha+1}$-closed and thus intersects $G^{n}\left(i_{\alpha+1}\right)$. 


\section{$5 \quad$ Generic for low complexity}

Here, the underlying forcing for each regular cardinal is an iteration and we have globally iterated products of these iterations. Forming a generic for this global iteration will be similar to the construction for the high complexity case, but more complicated. In this case, we can find a filtration $\left\{M_{n}^{i}: n<\omega\right\}$ for the local forcing at $i$, but the restriction of the local forcing at $i$ to these $M_{n}^{i}$ is not simply a regular subforcing.

Theorem $5.1\left(0^{\#}\right)$. Assume that $P_{\infty}=\langle P(\alpha): \alpha \in \mathrm{Ord}\rangle$ is the iteration with Easton support definable in $L$ without parameters as in Section 3 above. Then there exists $G$ which is $P_{\infty}$-generic over $L$.

Proof As before, we will define $G(\leq i)$ generic for $P(\leq i)$ by induction on $i \in I$. Let $i<j$ be adjacent indiscernibles in $I$. The only difference in the proof from the previous one will be the case of building a generic for $G(j)$ assuming that we have built $G(<j)$. Again, we will build $G(j)$ such that $\pi_{i, j}[G(i)] \subseteq G(j)$. (Note that, as before, we abuse the notation $\pi_{i, j}$ to mean the canonical extension of the indiscernible shift to the model $L[G(<i)]$.)

Define $\left\{M_{n}^{i}: n<\omega\right\}$ and $\left\{M_{n}^{j}: n<\omega\right\}$, filtrations at $i$ and $j$ respectively, in a canonical way as before such that $\pi_{i, j}\left(M_{n}^{i}\right)=M_{n}^{j}$ for each $n$. That is, let $M_{n}^{i}$ be the $\Sigma_{1}$ Hull of $i$ together with the next $n$ indiscernibles. Recall that each $M_{n}^{i}$ is in $L[G(\leq i)]$, but the sequence $\left\langle M_{n}^{i}: n<\omega\right\rangle$ is not.

As before, we would like to define the restriction of a condition to one of these models. Before we can do that, we will see that we can extend any condition such that all of its components have ground model names.

For each $p \in P(i)$, we can write $p=\langle p(\alpha): \alpha \in \operatorname{Dom}(p)\rangle$. We say that $p$ is self-determined if it has the property that for every $\alpha$ in the support of $p$, $p\left\lceil\alpha\right.$ forces $p(\alpha)=(\delta, X, \mathcal{A}, \mathcal{Z}, \Phi)$ as well as all $Z_{\gamma}\lceil\delta$, for $\gamma \in \mathcal{Z}$ to equal a name in the ground model $L[G(<i)]$.

It is dense for conditions in $P(i)$ to be self-determined since $p(\alpha)$ and $Z_{\gamma} \uparrow \delta$ for $\gamma$ mentioned in $p(\alpha)$ all have size $<i$ and therefore, we may extend any condition to be self-determined using the weak $i$-closure. Therefore, we will concentrate on conditions which have this property. 
Let $P^{*}(i)$ denote the set of conditions in $P(i)$ which are self-determined. We will define the restriction of $p \in P^{*}(i)$ to the model $M_{n}^{i}$ which we will denote as $p\left\lceil M_{n}^{i}\right.$. For $p \in P^{*}(i)$ let $p\left\lceil M_{n}^{i}=\left\{p(\alpha) \uparrow M_{n}^{i}: \alpha \in \operatorname{Dom}(p) \cap M_{n}^{i}\right\}\right.$ where $p(\alpha) \uparrow M_{n}^{i}$ is the obvious restriction of $p(\alpha)$ to the ordinals of $M_{n}^{i}$. Note that this restriction is an element of $M_{n}^{i}$ as $M_{n}^{i}$ is $i$-closed. We must see that this is still a condition and that extensions of it inside $M_{n}^{i}$ are compatible with $p$.

Claim 5.2. For all self-determined $p \in P(i)$, all $n<\omega$ and $\alpha \in \operatorname{Dom}(p) \uparrow M_{n}^{i}$, we have that

- $(p \uparrow \alpha) \uparrow M_{n}^{i}$ is a condition and

- if $q \in M_{n}^{i}$ is such that $q$ extends $\left(p\lceil\alpha) \uparrow M_{n}^{i}\right.$, then $p\lceil\alpha$ and $q$ are compatible.

Proof Let $\left\langle\alpha_{k}: k<\operatorname{ordertype}\left(\operatorname{Dom}(p) \cap M_{n}^{i}\right)\right\rangle$ be the enumeration of $\operatorname{Dom}(p) \cap M_{n}^{i}$ in increasing order. We will prove the claim by induction on $k$, first at successor stages and then at limit $k$.

Assume that $p$ is self-determined, that $\left(p\left\lceil\alpha_{k}\right) \uparrow M_{n}^{i}\right.$ is a condition and if $q \in M_{n}^{i}$ is such that $q$ extends $\left(p\left\lceil\alpha_{k}\right) \uparrow M_{n}^{i}\right.$, then $q$ is compatible with $p\left\lceil\alpha_{k}\right.$. We will first show that $\left(p\left\lceil\alpha_{k+1}\right) \uparrow M_{n}^{i}\right.$ is a condition.

The only reason that $\left(p \nmid \alpha_{k+1}\right) \uparrow M_{n}^{i}$ could fail to be a condition is if it fails to force $p\left(\alpha_{k}\right) \uparrow M_{n}^{i}$ to be a condition. So extend $\left(p\left\lceil\alpha_{k}\right) \uparrow M_{n}^{i}\right.$ to a condition $q$ which forces $p\left(\alpha_{k}\right) \uparrow M_{n}^{i}$ to not be a condition. We may assume that $q$ belongs to $M_{n}^{i}$. However, by the induction hypothesis, $q$ is compatible with $p\left\lceil\alpha_{k}\right.$ which forces $p\left(\alpha_{k}\right)$ to be a condition, contradiction.

To show the second part of the claim for $\alpha_{k+1}$, suppose that $\left(p\left\lceil\alpha_{k+1}\right) \uparrow M_{n}^{i}\right.$ is extended to a condition $q \in M_{n}^{i}$. We will show that there exists a condition $r$ which extends both $q$ and $p\left\lceil\alpha_{k+1}\right.$. Let $\operatorname{Dom}(r)=\operatorname{Dom}\left(p\left\lceil\alpha_{k+1}\right) \cup \operatorname{Dom}(q)\right.$. First we will consider $\alpha \in \operatorname{Dom}\left(p\left\lceil\alpha_{k+1}\right) \cap \operatorname{Dom}(q)\right.$. Since $\operatorname{Dom}\left(p\left\lceil\alpha_{k+1}\right) \cap\right.$ $\operatorname{Dom}(q)=\operatorname{Dom}\left(\left(p\left\lceil\alpha_{k+1}\right) \uparrow M_{n}^{i}\right)\right.$, we know that $\delta_{q(\alpha)} \geq \delta_{p(\alpha)}$ We can take $r(\alpha)=q(\alpha) \cup p(\alpha)$ where

1. $\mathcal{A}_{r(\alpha)}=\mathcal{A}_{q(\alpha)} \cup \mathcal{A}_{p(\alpha)}$,

2. $\mathcal{Z}_{r(\alpha)}=\mathcal{Z}_{q(\alpha)} \cup \mathcal{Z}_{p(\alpha)}$, 
3. $\delta_{r(\alpha)}$ is a large enough ordinal $<i$ such that ordinals in $\left[\delta_{q(\alpha)}, \delta_{r(\alpha)}\right)$ can be written as the disjoint union of $\left|\mathcal{Z}_{r(\alpha)}\right|$-many copies of $\left[\delta_{p(\alpha)}, \delta_{r(\alpha)}\right)$,

4. $\Phi_{r(\alpha)}$ extends $\Phi_{q(\alpha)} \cup \Phi_{p(\alpha)} \uparrow\left(\operatorname{Dom}\left(\Phi_{p(\alpha)}\right)-M_{n}^{i}\right)$ such that for all $\gamma \in \mathcal{Z}_{q(\alpha)}, \Phi_{r(\alpha)} \operatorname{maps} Z_{\gamma} \uparrow\left[\delta_{q(\alpha)}, \delta_{r(\alpha)}\right)$ into one of the copies of $\left[\delta_{p(\alpha)}, \delta_{r(\alpha)}\right)$ above $\delta_{q(\alpha)}$ and for all $\gamma \in \mathcal{Z}_{p(\alpha)}, \Phi_{r(\alpha)}$ maps $Z_{\gamma} \uparrow\left[\delta_{p(\alpha)}, \delta_{r(\alpha)}\right)$ into one of the copies of $\left[\delta_{p(\alpha)}, \delta_{r(\alpha)}\right)$ above $\delta_{q(\alpha)}$,

5. $X_{r(\alpha)}=X_{q(\alpha)} \cup\left[\delta_{q(\alpha)}, \delta_{r(\alpha)}\right)$. The extra relations on $X_{r(\alpha)}$ connect the images of $Z_{\gamma} \uparrow \delta_{p(\alpha)}$ with those of $Z_{\gamma} \uparrow\left[\delta_{p(\alpha)}, \delta_{r(\alpha)}\right)$ in the same way as $Z_{\gamma}$ for all $\gamma \in \mathcal{Z}_{p(\alpha)}$, and likewise for $\gamma \in \mathcal{Z}_{q(\alpha)}$.

If $\alpha \in \operatorname{Dom}(r) \backslash \operatorname{Dom}\left(p\left\lceil\alpha_{k+1}\right)\right.$, then let $r(\alpha)=q(\alpha)$ and if $\alpha \in \operatorname{Dom}(r) \backslash \operatorname{Dom}(q)$, then let $r(\alpha)=p(\alpha)$.

Since the components inside and outside the model $M_{n}^{i}$ are disjoint and $X_{q} \uparrow \delta_{p}=X_{p}$, the unions will cause no conflict. The rest of the argument that this is a condition follows from [9, proof of Theorem 4, Claims 2 and 3].

If $k$ is a limit ordinal, then assume for all $l<k$, we have $\left(p\left\lceil\alpha_{l}\right) \uparrow M_{n}^{i}\right.$ is a condition and if $q \in M_{n}^{i}$ is such that $q$ extends $\left(p\left\lceil\alpha_{l}\right) \uparrow M_{n}^{i}\right.$, then $q$ is compatible with $p\left\lceil\alpha_{l}\right.$. To see that $\left(p\left\lceil\alpha_{k}\right) \uparrow M_{n}^{i}\right.$ is a condition, we only need to note that the restriction operations are canonical, that is, for $l<m<k$ we have $\left(\left(p\left\lceil\alpha_{m}\right) \uparrow M_{n}^{i}\right) \uparrow \alpha_{l}=\left(p\left\lceil\alpha_{l}\right) \uparrow M_{n}^{i}\right.\right.$.

Finally, we need to show that if $q$ extends $\left(p\left\lceil\alpha_{k}\right) \uparrow M_{n}^{i}\right.$, then $q$ is compatible with $p\left\lceil\alpha_{k}\right.$. We may construct $r$ extending $\left(p\left\lceil\alpha_{k}\right) \uparrow M_{n}^{i}\right.$ and $q$ as before letting $\operatorname{Dom}(r)=\bigcup_{l<k} \operatorname{Dom}\left(p\left\lceil\alpha_{l}\right) \cup \operatorname{Dom}(q)\right.$.

Claim 5.3. If $p \in P^{*}(i)$ and $q \in M_{n}^{i}$ such that $q$ extends $p\left\lceil M_{n}^{i}\right.$, then $p$ and $q$ are compatible.

Proof By Claim 5.2, we know that $p \nmid M_{n}^{i}$ is a condition. Construct $r$ extending $p$ and $q$ exactly as in Claim 5.2, letting $\operatorname{Dom}(r)=\operatorname{Dom}(p) \cup \operatorname{Dom}(q)$.

Let $G^{*}(i)=G(i) \cap P^{*}(i)$. We have sufficiently prepared the conditions in $G^{*}(i)\left\lceil M_{0}^{i}\right.$ in order to shift them up to $P(j)$. Let $A_{0}=\left\{\pi_{i, j}\left(p\left\lceil M_{0}^{i}\right): p \in\right.\right.$ $\left.G^{*}(i) \cap M_{0}^{i}\right\}$. 
Since each condition $p$ has size $<i$, this is also true of each condition in $A_{0}$. We know that $\left|A_{0}\right| \leq i$ since $\left|M_{0}^{i}\right|=i$. We may take the union of the conditions in $A_{0}$, call this $p_{0}^{0}$. By [9, proof of Theorem 4, Claim 5], this is a condition in $P(j)$. Using the weak $j$-closure of $P(j)$, we may extend $p_{0}^{0}$ to $p_{0}^{1} \in P(j) \cap M_{0}^{j}$ hitting $<j$-many dense sets in $M_{0}^{j}$.

We want to continue this at each $M_{n}^{j}$ for $n<\omega$. We will do this by constructing the master condition at $M_{n}^{j}$ and for each $m \leq n$ we will extend the conditions built before to hit even more (but less than $j$ many) dense sets in $M_{m}^{j}$. Since in $L\left[0^{\#}\right]$ we have $\operatorname{cf}(j)=\omega$, at the end of the construction for each $m$ we will have a condition which hits all dense sets in $M_{m}^{j}$.

After step $n$ we formed $p_{m}^{n-m}$ for $m \leq n$ as above. Extend each $p_{m}^{n-m}$ to $p_{m}^{(n-m)+1}$, hitting $<j$-many dense sets in $M_{m}^{j}$. Form $p_{n+1}^{0}$ as the union of conditions in $A_{n+1}=\left\{\pi_{i, j}\left(p\left\lceil M_{n+1}^{i}\right): p \in G^{*}(i) \cap M_{n+1}^{i}\right\}\right.$. We know that $p_{n+1}^{0}$ is compatible with $p_{m}^{(n-m)+1}$ by Claim 5.3. Extend $p_{n+1}^{0}$ to $p_{n+1}^{1} \leq p_{m}^{(n-m)+1}$ for all $m \leq n$ such that $p_{n+1}^{1}$ hits $<j$-many dense sets in $M_{n+1}^{j}$.

The generic $G(j)$ will then be $\left\{p \in P(j): p \geq p_{n}^{m}\right.$ : for some $\left.m, n<\omega\right\}$.

\section{Conclusion}

As remarked in the introduction, in the inductive process of building generics for reverse Easton class forcings, it is often not possible to simply modify any generic to cohere with what was built before. The results in sections 4 and 5 give techniques for finding generics for reverse Easton iterations even when this modification is not possible.

To continue with the programme of deciding when such forcings can have generics, the question remains: what intrinsic properties of the given forcings are used to allow these techniques to work? Below we isolate the main attributes of a global forcing which are sufficient to use the techniques of sections 4 and 5 .

Theorem 6.1. If $P_{\infty}=\langle P(\alpha): \alpha \in$ Ord $\rangle$ is a class forcing with Easton 
support which is $L$-definable without parameters and the following properties hold, then there exist a generic for $P_{\infty}$ over $L$.

1. For all $\alpha \in$ Ord we have $P(\alpha)$ has the $\alpha$-weak closure.

2. For $i \in I$ let $G(i)$ be generic for $P(i)$ and let $\left\langle M_{n}^{i}: n<\omega\right\rangle$ be the sequence of elementary submodels of $L[G(<i)]$ defined by $M_{n}^{i}=$ $\Sigma_{1} \operatorname{Hull}\left(i \cup \vec{\infty}_{n}\right)$ where $\vec{\infty}_{n}$ are the first $n$ indiscernibles greater than $i$. (Each $M_{i}^{n}$ is $i$-closed and has size $i$.) For an indiscernible $j>i$, let $\left\langle M_{n}^{j}: n<\omega\right\rangle$ be the corresponding sequence of elementary submodels of $L[G(<j)]$. Note that $\pi_{i, j}\left(M_{n}^{i}\right)=M_{n}^{j}$.

For any $P_{\infty}$-generic $G$ there are sequences $\left\langle D_{\alpha}: \alpha \in \mathrm{Ord}\right\rangle,\left\langle\uparrow_{\alpha}: \alpha \in \mathrm{Ord}\right\rangle$, $\left\langle m c_{\alpha}: \alpha \in \operatorname{Ord}\right\rangle$ where $D_{\alpha}, \Gamma_{\alpha}$ and $m c_{\alpha}$ are definable in $L[G(<\alpha)]$ uniformly (and independently of $G$ ) such that for each $i \in I$ :

(a) $D_{i}$ is dense in $P(i)$.

(b) For $p \in D_{i}$ and $M$ an $i$-closed elementary submodel of $L[G(<i)]$ of size $i, p \uparrow_{i} M$ is a condition in $P(i)$ such that for all $q$ extending $p \uparrow_{i} M$, if $q \in M$ then $q$ is compatible with $p$.

(c) For $i<j, i, j \in I$ and for each $n<\omega, m c\left(\pi_{i, j}\left[G(i) \cap M_{n}^{i}\right]\right)$ is a lower bound for the conditions in $\pi_{i, j}\left[G(i) \cap M_{n}^{i}\right]$; moreover, for any $n_{0}<n, m c\left(\pi_{i, j}\left[G(i) \cap M_{n}^{i}\right]\right) \uparrow M_{n_{0}}^{j}=m c\left(\pi_{i, j}\left[G(i) \cap M_{n_{0}}^{i}\right]\right)$.

Note that in the proofs in sections 4 and 5, property (1) was used to build generics between indiscernibles and property (2) was used to construct a generic at a successor indiscernible stage which coheres with the generics at previous indiscernible stages.

If we restrict our attention to a natural class of product forcings, we can get a result which is more easily verifiable.

Definition 6.2. A product forcing $P(\alpha)$ is internally small iff the support of $P$ has size $<\alpha$ and each component of the product is a forcing which has size $\alpha$.

Note that we do not say that $P(\alpha)$ is simply "small" as this would imply that the length of the product is also restricted. We do not require this. 
Theorem 6.3. If $P_{\infty}=\langle P(\alpha): \alpha \in$ Ord $\rangle$ is a class forcing with Easton support which is $L$-definable without parameters and the following properties hold, then there exist a generic for $P_{\infty}$ over $L$.

1. $P(\alpha)$ is a internally small product forcing.

2. For all $\alpha \in$ Ord we have $P(\alpha)$ has $\alpha$-closure.

Note that here we require full $\alpha$-closure whereas the product forcing notion introduced in section 2 only has weak closure. In general weak closure is not enough to shift up conditions in the generic restricted to a model from an indiscernible $i$ to a greater indiscernible $j$ and get a master condition as in 2(c) of Theorem 6.1. In particular, the lower bounds for the sequences in the generic which satisfy a weak closure operation may not be in the generic. In section 4 , we do get such a master condition, but this requires a proof which is particular to our case.

\section{References}

[1] A. Beller, R. Jensen, and P. Welch. Coding the universe, volume 47 of London Mathematical Society Lecture Note Series. Cambridge University Press, Cambridge-New York, 1982.

[2] N. Dobrinen and S. Friedman. Internal consistency and co-stationarity of the ground model. accepted in the Journal of Symbolic Logic. http://www.logic.univie.ac.at/ sdf/papers/.

[3] M. Džamonja, S. Friedman, and K. Thompson. Global complexity results. In A. Andretta, editor, Set Theory: Recent Trends and Applications, volume 17 of Quaderni di Matematica, pages 25-45. Seconda Universita di Napoli, 2007.

[4] W.B. Easton. Powers of regular cardinals. Annals of Mathematical Logic, $1,1970$. 
[5] S. Friedman. Fine structure and class forcing. de Gruyter, 2000.

[6] S. Friedman. Internal consistency and the inner model hypothesis. Bulletin of Symbolic Logic, 12(4):591-600, 2006.

[7] S. Friedman and P. Ondrejović. Internal consistency of Easton's theorem. submitted. http://www.logic.univie.ac.at/ sdf/papers/.

[8] S. Friedman and K. Thompson. Perfect trees and generic modification. submitted. http://www.logic.univie.ac.at/ thompson/pubs.html.

[9] P. Komjáth and S. Shelah. Universal graphs without large cliques. Journal of Combinatorial Theory, 63(1):125-135, 1995.

Sy-David Friedman

sdf@logic.univie.ac.at

Kurt Gödel Research Center for Mathematical Logic

University of Vienna

Austria

Katherine Thompson

aleph_nought@yahoo.com

Kurt Gödel Research Center for Mathematical Logic

University of Vienna

Austria 